\title{
Correction to: In Vivo Stability of Therapeutic Proteins
}

Joachim Schuster ${ }^{1,2}$ - Atanas Koulov' - Hanns-Christian Mahler ${ }^{\prime}$ Pascal Detampel ${ }^{2}$. Joerg Huwyler ${ }^{2} \cdot$ Satish Singh' $\cdot$ Roman Mathaes'

Published online: 12 March 2020

(C) Springer Science+Business Media, LLC, part of Springer Nature 2020

\section{Correction to: Pharmaceutical Research}

https://doi.org/10.1007/s1 1095-019-2689-1

A manuscript version without peer-review revisions was mistakenly processed and published. The original article has been corrected.

The online version of the original article can be found at https://doi.org/l0. |007/s | |095-0|9-2689-|

$\triangle$ Roman Mathaes

roman.mathaes@lonza.com

Drug Product Services, Lonza AG, Basel, Switzerland

2 Division of Pharmaceutical Technology, University of Basel,

Basel, Switzerland 